\section{Fatores associados à coinfecção tuberculose e HIV: o que apontam os dados de notificação do Estado do Amazonas, Brasil, 2001-2012}

\author{
Factors associated with TB/HIV coinfection: \\ evidence from notification data in the State \\ of Amazonas, Brazil, 2001-2012
}

Evela da Silva Magno 1

Valeria Saraceni 2

Alexandra Brito de Souza 1

Regma da Silva Magno 3

Maria das Graças Gomes Saraiva 1

Samira Bührer-Sékula 1,4

doi: 10.1590/0102-311X00019315

\title{
Resumo
}

A tuberculose (TB) em pessoas vivendo com HIV (PVHIV) é a maior causa infecciosa de morte relacionada à AIDS. O objetivo deste trabalho foi estimar a prevalência da coinfecção TB/HIV, avaliar a notificação dos dois agravos de maneira temporal, por meio de relacionamento de bases de dados, e buscar fatores associados às notificações de AIDS e TB. A prevalência de coinfecção TB/HIV foi de 7,7\%. O grupo de PVHIV com posterior diagnóstico de TB foi o mais representativo, apesar de medidas preventivas disponíveis. A subnotificação de TB entre os casos de AIDS foi de 35\%, e 19,6\% dos casos de TB poderiam ser notificados como AIDS. Para os casos de AIDS com menção de $T B$, residir na capital apresentou uma chance $75 \%$ maior de ser notificado no Sistema de Informação de Agravos de Notificação/Tuberculose (SINAN TB), e ter falecido aumentou em 40\% a chance de registro. Dos casos de TB com menção de HIV, ser de cor parda, ter entre 25 e 39 anos, residir na capital e ter evoluído para o óbito estiveram associados à maior chance de notificação na AIDS. O linkage periódico dessas bases pode ser uma ferramenta programática poderosa para diminuir a subnotificação.

Notificação de Doenças; Infecções Oportunistas; Tuberculose; HIV

\author{
Correspondência \\ S. Bührer-Sékula \\ Instituto de Patologia Tropical e Saúde Pública, Universidade \\ Federal de Goiás. \\ Rua 235 s/n, Goiânia, GO 74605-050, Brasil. \\ samira@buhrer.net \\ 1 Fundação de Medicina Tropical Dr. Heitor Vieira Dourado, \\ Manaus, Brasil. \\ 2 Secretaria Municipal de Saúde do Rio de Janeiro, Rio de \\ Janeiro, Brasil. \\ 3 Universidade Nilton Lins, Manaus, Brasil. \\ 4 Instituto de Patologia Tropical e Saúde Pública, Universidade \\ Federal de Goiás, Goiânia, Brasil.
}




\section{Introdução}

A infecção pelo HIV aumenta o risco de desenvolvimento de tuberculose (TB) ativa em 20 vezes, tanto por reativação de infecção latente pelo Mycobacterium tuberculosis (ILTB) como por reinfecção 1. Para diminuir o risco de desenvolvimento de TB em pessoas vivendo com HIV (PVHIV), combinado ao diagnóstico precoce de HIV, é preconizada a utilização de antirretrovirais (ART) para o tratamento da infecção pelo HIV 2,3 e o tratamento da ILTB com isoniazida 4,5,6 quando as PVHIV apresentarem prova tuberculínica (PPD) com área de induração maior ou igual a $5 \mathrm{~mm}$, naquelas com uma cicatriz compatível com TB na telerradiografia de tórax e sem história de tratamento anterior, ou ainda, no caso dos contatos de pacientes com TB bacilífera 7,8 .

O Estado do Amazonas é a unidade da federação com a maior incidência de TB no Brasil, com 68,4 casos novos por 100 mil habitantes ${ }^{9}$, posto que já foi ocupado pelo Estado do Rio de Janeiro. Entretanto, apesar de diferenças no comportamento das epidemias de HIV/AIDS nos dois estados, estudos realizados no Amazonas e no Rio de Janeiro apontam a TB como importante causa de morte 10,11.

Até 2013, a AIDS seguiu critérios para notificação dos casos que dependiam de doenças indicativas de imunodeficiência, contagem de células T-CD4+ abaixo de 350células $/ \mathrm{mm}^{3}$, ou indivíduos que morriam pela doença 12, o que limitava a notificação a casos de AIDS. Em 2014, a infecção pelo HIV se tornou um agravo de notificação compulsória no país 13 .

No presente trabalho a prevalência da coinfecção TB/HIV/AIDS foi estimada e a notificação dos dois agravos avaliada de maneira temporal nas PVHIV, assim como identificados os fatores associados à notificação de AIDS e TB, no Estado do Amazonas, no período de 2001 a 2012.

\section{Material e métodos}

Os dados de notificação de TB e AIDS, em maiores de 13 anos, no Sistema de Informação de Agravos de Notificação (SINAN) e os dados de mortalidade do Sistema de Informações sobre Mortalidade (SIM) foram obtidos na Fundação de Vigilância em Saúde do Estado do Amazonas (FVS-AM), por meio das Coordenações de TB e DST/AIDS.

Na base de AIDS, os casos informados como portadores de TB, no momento da notificação dentro do critério Rio de Janeiro/Caracas, foram selecionados como casos de coinfecção AIDS/TB a priori, formando um grupo específico para a evolução temporal dos casos em relação ao diagnóstico das duas doenças.

Valendo-se da base de dados da TB foi possível estabelecer os casos com HIV/AIDS no momento da notificação de TB, por meio da variável HIV.

A data do diagnóstico de HIV foi definida a partir das datas existentes na notificação de AIDS, envolvendo as datas do teste de triagem, do teste confirmatório, do teste rápido ou do diagnóstico, nesta ordem. A data de diagnóstico de TB foi assumida como a de início do tratamento específico. Com essas datas foi possível estabelecer a relação temporal entre os dois eventos.

Para gerar o linkage das bases foi necessário ter acesso aos nomes dos indivíduos. Para tanto, foi utilizado o Termo de Compromisso de Utilização de Dados e os procedimentos deste estudo foram aprovados pelo Comitê de Ética em Pesquisa da Fundação de Medicina Tropical Dr. Heitor Vieira Dourado, em 5 de outubro de 2012, aprovação no 121.958, conforme a Resolução no 466/2012 do Conselho Nacional de Saúde.

\section{Etapa de pareamento}

No Estado do Amazonas, no período de 2001 a 2012, foram notificados 6.753 casos de AIDS, sendo que em 13,7\% (926/6.753) dos casos a notificação fazia menção à TB. No mesmo período foram notificados 30.860 casos de TB, com 6,7\% (2.059/30.860) de menções de HIV/AIDS.

Para realizar o relacionamento probabilístico das bases de dados por meio do software RecLink 14, os registros das duas bases foram padronizados: registros duplicados foram eliminados manualmente analisando-se nome do paciente, data de nascimento e nome da mãe usando-se o Excel (Microsoft Corp., Estados Unidos). Os parâmetros utilizados para o linkage foram os sugeridos por Camargo Jr. 
\& Coeli 14, a saber: "nome" - tipo "aproximado" com: correto: 90\%, incorreto: 10\%, limiar: 85\%; "data" - tipo: caractere com: correto: 90\%, incorreto: 10\%, limiar: 65\%; e "sexo" - tipo exato com” correto: 90\%, incorreto: $50 \%$, sem utilização de limiar. O valor mínimo do escore para inclusão foi de 2,0.

O linkage foi realizado em duas etapas, a primeira envolvendo o SINAN AIDS (base principal para o linkage) com o SINAN TB, usando-se "nome", "sexo" e "data do nascimento". Os passos de blocagem foram três, o primeiro com "nome", "sexo" e "data do nascimento"; o segundo com "nome" e "data do nascimento"; e o terceiro com "nome" e "sexo". A filiação materna de todos os pares encontrados foi checada manualmente, independentemente do escore, gerando uma base formada por casos que estavam presentes tanto no SINAN AIDS como no SINAN TB (grupo de pares). Esses pares foram separados em quatro grupos, a saber: grupo 1 (G1) - o diagnóstico de TB precedeu o de HIV; grupo 2 (G2) - os diagnósticos de TB e HIV aconteceram de maneira simultânea, como na testagem anti-HIV dos pacientes com TB durante o tratamento desta ou na triagem de TB em pacientes recém-diagnosticados com HIV; grupo 3 (G3) - o diagnóstico de HIV precedeu o da TB; e grupo 4 (G4) - casos notificados de AIDS com menção de TB.

Na segunda etapa, o linkage foi realizado entre a base de dados do SIM incluindo todos os pacientes que não formaram pares ("par não encontrado"), ou seja: (1) pacientes que estavam no SINAN TB mas não no SINAN AIDS; e (2) pacientes que estavam no SINAN AIDS com diagnóstico de TB mas não estavam no SINAN TB, mais os grupos de pares G2, G3 e G4. Foi excluído o G1 porque a TB ocorreu antes do HIV. Os casos de TB e/ou HIV encontrados no SIM foram considerados como óbitos, independentemente da causa básica de óbito declarada.

\section{Análises estatísticas}

A relação temporal entre os dois eventos foi descrita levando-se em consideração se os casos foram ou não encontrados no linkage AIDS/TB. Os grupos decorrentes foram comparados em relação às variáveis sociodemográficas e as relativas às duas doenças. Foi usado o teste de qui-quadrado de Pearson para avaliar a homogeneidade de proporções de variáveis categóricas, e os testes t de Student ou de Wilcoxon para comparar médias ou medianas de variáveis contínuas.

A variável raça/cor com múltiplas categorias gerou uma variável binária (cor parda, sim/não), por conta da distribuição em pequenos números em algumas categorias, como amarela e indígena.

Para identificar fatores associados à notificação de casos de TB e AIDS nas duas bases de dados, procedeu-se a uma regressão logística uni e multivariada comparando os casos identificados nas duas bases (pares e não pares), tendo como desfecho a notificação no SINAN TB, e como variáveis independentes as características sociodemográficas e clínicas dos casos. Para entrar no modelo multivariado final de regressão, as variáveis independentes com $\mathrm{p}<0,20$ foram selecionadas e para permanecer no modelo trabalhou-se com uma significância de 5\%. Os dados foram analisados no pacote estatístico Stata, versão 11.2 (StataCorp LP, College Station, Estados Unidos).

Como estratégia para identificar a subnotificação de casos de AIDS lançou-se mão dos critérios Rio de Janeiro/Caracas, em que os casos de TB declarados como extrapulmonares ou disseminados, assim como os que atendiam ao critério óbito, deveriam ter sido notificados na base de AIDS. Já para a TB, a subnotificação foi quantificada de maneira imediata entre os casos encontrados na base AIDS com menção de TB e sem a respectiva notificação na base de TB, já que esta seria compulsória.

\section{Resultados}

Os escores resultantes variaram de 2,845 a 7,188. O linkage das bases SINAN AIDS e SINAN TB resultou em 1.469 pares de casos que haviam sido notificados nas duas bases, destes, 15\% não tinham resultado de HIV na ficha de notificação de TB. Dos pares, em 6,7\% (98) dos casos a TB precedeu o diagnóstico de HIV (G1), em 8,9\% (131) o diagnóstico de TB e HIV foi simultâneo (G2), em 43,5\% (639) o diagnóstico de HIV precedeu o de TB (G3), e em 40,9\% (601) dos casos a TB foi assinalada no momento da notificação de AIDS (G4) (Figura 1a).

Para criar o grupo de comparação de não pares na notificação das duas doenças, dos 926 casos sabidos de coinfecção na base da AIDS subtraiu-se 601 de AIDS com marcação de TB (G4), resultando 
em 325 casos de HIV/AIDS não reportados no SINAN TB. Esses casos representam uma subnotificação de TB de 35\% (325/926) ente esses casos de AIDS, já que todos os casos de TB são de notificação compulsória (Figura 1b).

Dos 2.059 casos notificados de TB com a variável HIV com resposta igual a positivo na base TB, foram subtraídos os 1.225 também notificados no SINAN AIDS, resultando em 834 casos de TB/HIV sem notificação no SINAN AIDS. Desses, 307 casos de TB extrapulmonar deveriam ter sido notificados como AIDS, por preencherem o critério Rio de Janeiro/Caracas e, também, 97 casos pulmonares que faleceram e atendiam ao critério óbito, totalizando 19,6\% (404/2.059) de subnotificações de AIDS entre os casos de TB, de acordo com os critérios de notificação de AIDS no Brasil à época (Figura 1c).

Quando adicionamos os 325 casos de AIDS com menção de TB não registrados no SINAN TB aos 2.059 já declarados na mesma base, a prevalência de coinfecção aumentou de 6,7\% para 7,6\%.

\section{Comparação das características dos grupos}

Comparando-se o grupo com diagnóstico de TB e HIV simultâneo (G2) com o grupo em que a TB estava presente na notificação de AIDS (G4), os grupos diferiram na distribuição proporcional da categoria de transmissão $(\mathrm{p}=0,03)$ e em relação à proporção de óbitos $(40,5 \%$ vs. $30,9 \%, \mathrm{p}=0,035)$ (Tabela 1).

Na Tabela 2, está a comparação entre os grupos G3, em que o diagnóstico de TB ocorreu posteriormente ao de HIV, e G4, no qual a TB estava informada na notificação de AIDS. Os grupos diferiram na distribuição proporcional da faixa etária $(\mathrm{p}=0,001)$ e da categoria de transmissão $(\mathrm{p}<0,001)$.

Excluindo-se o G1, que apresentou TB antes do diagnóstico de HIV, foi realizado o linkage com o SIM e, dos 1.371 casos considerados como coinfecção TB/HIV/AIDS (G2, G3 e G4), 31,1\% (426/1.371) eram casos de óbito.

Na Tabela 3, observamos que entre os casos de TB notificados no SINAN AIDS e ausentes do SINAN TB a residência na capital apresentou uma chance $75 \%$ maior de ser notificada no SINAN TB (OR $=$ 1,75; IC95\%: 1,15-2,66), e ter falecido em decorrência da TB aumentou em $40 \%$ a chance (OR = 1,40; IC95\%: 1,05-1,87) de registro. O modelo final multivariado mostrou que, controlando por sexo e idade, residir na capital apresentou uma OR ajustada de 1,73 (IC95\%: 1,13-2,65), e ter evoluído para o óbito uma OR = 1,41 (IC95\%: 1,05-1,88), ambas com significância estatística.

Para os casos não notificados no SINAN AIDS, porém notificados no SINAN TB e com a variável HIV preenchida como positiva, observa-se na Tabela 4 que ser de cor parda $(\mathrm{OR}=1,52$; IC95\%: 1,231,87), estar na faixa etária de 25 a 39 anos ( $\mathrm{OR}=1,33$; IC95\%: 1,02-1,73), residir na capital (OR = 2,02; IC95\%: 1,56-2,62) e ter evoluído para o óbito ( $\mathrm{OR}=1,67 ; \mathrm{IC} 95 \%$ : 1,36-2,06) foram associados à maior chance de notificação de AIDS. No modelo final multivariado, controlado por sexo, as mesmas variáveis permaneceram significativas.

\section{Discussão}

Utilizar as bases de dados do SINAN TB e AIDS e o relacionamento entre as bases para estimar a real prevalência da coinfecção tem sido uma ferramenta útil para monitorar a ocorrência do evento TB/ HIV, uma vez que a subnotificação destas doenças pode ser um limitador da estimativa de coinfecção. Neste estudo, calculando-se a coinfecção pelas duas bases de dados separadamente, a prevalência de coinfecção TB/HIV foi inicialmente de 6,7\% entre os 30.860 notificados no SINAN TB e 13,7\% entre os 6.753 notificados no SINAN AIDS. Tradicionalmente, refere-se como coinfecção TB/HIV os casos notificados de TB no SINAN TB que apresentam a variável HIV marcada como positiva. Dentro dessa definição, a coinfecção estimada para o Brasil entre 2000 e 2005 foi de 6,9\% (35.728 notificados corretamente) no SINAN TB, e a subnotificação de AIDS entre os casos de TB foi de 8,4\% (7.676 sem registro de HIV/AIDS entre 43.404 notificados) 15 . Comparando-se os estudos, a prevalência de coinfecção no Amazonas é semelhante à do Brasil, mas com a adição de casos não notificados na TB ultrapassou a média nacional $(7,7 \%$ vs. 6,9\%).

Baseando-se no SINAN AIDS encontramos uma subnotificação de TB de 35\%. Superior aos 17,7\% observados para o Brasil, no período de 2001 a 2005 15. Um estudo no Rio de Janeiro em 2007/2008, 


\section{Figura 1}

(1a) Pacientes notificados nos dois bancos (SINAN AIDS e SINAN TB) e distribuição dos pares por tipo de entrada definida pela relação temporal entre as doenças; (1b) Número de casos notificados no SINAN AIDS com menção de tuberculose (TB), mas não notificados no SINAN TB; (1c) Número de casos notificados no SINAN TB, mas não notificados no SINAN AIDS.

1a)

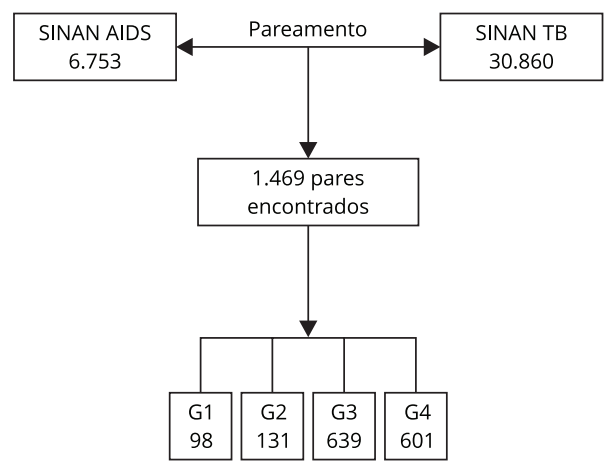

$1 b)$

SINAN AIDS

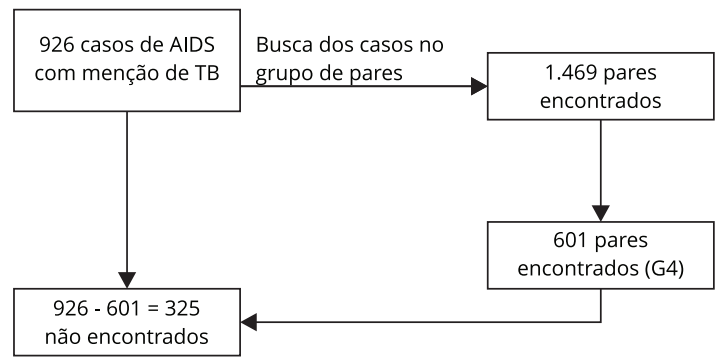

1c)

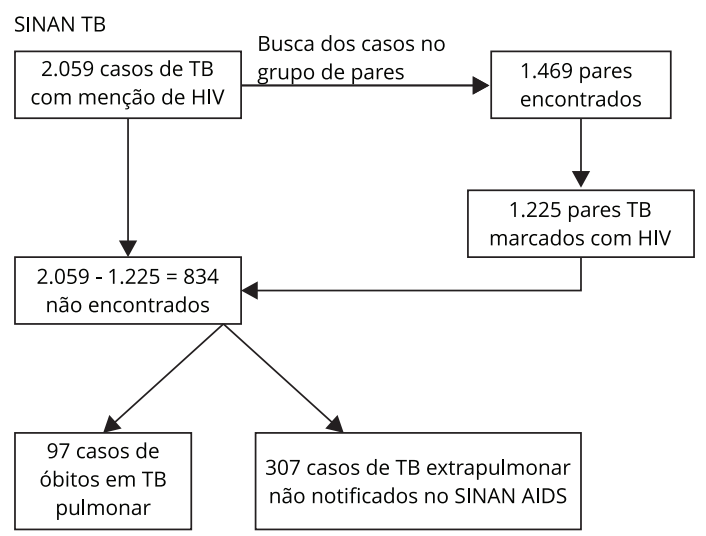

G1: diagnóstico de TB precedeu o de HIV; G2: diagnósticos de TB e HIV aconteceram de maneira simultânea; G3: diagnóstico de HIV precedeu o da TB; G4: casos notificados de AIDS com menção de TB; SINAN: Sistema de Informação de Agravos de Notificação. 
Tabela 1

Distribuição proporcional das características sociodemográficas e clínicas dos casos com diagnóstico simultâneo de tuberculose (TB) e HIV (G2) e com tuberculose marcada na notificação de AIDS (G4), Amazonas, Brasil, 2001 a 2012.

\begin{tabular}{|c|c|c|c|c|c|}
\hline \multirow[t]{2}{*}{ Características } & \multicolumn{2}{|c|}{ TB/HIV simultâneo (G2) } & \multicolumn{2}{|c|}{ TB na notificação AIDS (G4) } & \multirow[t]{2}{*}{ Valor de $p$} \\
\hline & $n=131$ & $\%$ & $n=601$ & $\%$ & \\
\hline \multicolumn{6}{|l|}{ Sexo } \\
\hline Masculino & 96 & 73,3 & 428 & 71,2 & 0,634 \\
\hline Feminino & 35 & 26,7 & 173 & 28,8 & \\
\hline \multicolumn{6}{|l|}{ Faixa etária (anos) } \\
\hline $13-24$ & 19 & 15,5 & 89 & 14,8 & 0,502 \\
\hline $25-40$ & 77 & 58,8 & 322 & 53,6 & \\
\hline$\geq 40$ & 35 & 26,7 & 190 & 31,6 & \\
\hline \multicolumn{6}{|l|}{ Raça/Cor } \\
\hline Outras & 21 & 16 & 98 & 16,3 & 0,938 \\
\hline Parda & 110 & 84 & 503 & 83,7 & \\
\hline \multicolumn{6}{|l|}{ Categoria de transmissão } \\
\hline Homossexual/Bissexual & 18 & 13,8 & 123 & 20,5 & 0,003 \\
\hline Heterossexual & 60 & 45,8 & 326 & 54,2 & \\
\hline Sangue/Droga intravenosa & 2 & 1,5 & 12 & 2,0 & \\
\hline Ignorado & 51 & 38,9 & 140 & 23,3 & \\
\hline \multicolumn{6}{|l|}{ Residência } \\
\hline Capital & 119 & 90,8 & 547 & 91,0 & 0,949 \\
\hline Interior & 12 & 9,2 & 54 & 9,0 & \\
\hline \multicolumn{6}{|l|}{ Forma da TB } \\
\hline Pulmonar & 75 & 57,3 & 356 & 59,2 & 0,676 \\
\hline Extrapulmonar & 56 & 42,7 & 245 & 40,8 & \\
\hline \multicolumn{6}{|l|}{ Óbito } \\
\hline Sim & 53 & 40,5 & 186 & 30,9 & 0,035 \\
\hline Não & 78 & 59,5 & 415 & 69,1 & \\
\hline
\end{tabular}

Fonte: Sistema de Informação de Agravos de Notificação (SINAN) e Sistema de Informações sobre Mortalidade (SIM)/Amazonas.

encontrou uma subnotificação de TB de 5,3\%, com base nos livros de registro e acompanhamento de casos de TB 16. Em um hospital em Pisa, Itália, o relacionamento de bases de dados de registros hospitalares e laboratoriais encontrou uma subnotificação de 27\% entre casos de TB confirmados 17 . Nesse mesmo estudo, os fatores associados à notificação foram ter TB cavitária, com baciloscopia e/ ou cultura positiva e idade mais baixa, enquanto que o presente trabalho revelou associação de notificação com a residência na capital (Manaus) e ter evoluído para o óbito. O Estado do Amazonas tem uma extensão territorial muito grande, com as cidades do interior com menor resolubilidade, o que pode explicar a concentração de notificação de casos na capital, com mais recursos na área de saúde. O relacionamento das bases de dados do SIM com o SINAN TB proporcionou a criação de uma lista de casos cujo desfecho foi óbito nos casos de TB, que muitas vezes poderiam ser considerados como abandono, para posterior correção no SINAN TB, e aumentou a frequência dos casos de AIDS no Estado do Amazonas em 11,8\%, superior aos 3,7\% observados no Brasil em 201218.

No Município do Rio de Janeiro, foi relatada uma subnotificação de AIDS de 42,7\% pelo relacionamento da base do SINAN AIDS com a do Sistema de Informações Hospitalares (SIH) em 199619. No presente trabalho, a subnotificação de AIDS pelo linkage das duas bases do SINAN encontrou um valor inferior, de 19,6\%. Em relação aos fatores associados à notificação de AIDS, nosso estudo encontrou a cor parda, a idade entre 25 e 39 anos, residir na capital e ter evoluído para óbito. Outro trabalho realizado no Rio de Janeiro não identificou associação de características sociodemográfi- 


\section{Tabela 2}

Distribuição proporcional das características sociodemográficas e clínicas dos casos com diagnóstico de tuberculose (TB) posterior ao de HIV (G3) e HIV com TB marcada na notificação de AIDS (G4). Amazonas, Brasil, 2001 a 2012.

\begin{tabular}{|c|c|c|c|c|c|}
\hline \multirow[t]{2}{*}{ Características } & \multicolumn{2}{|c|}{ TB posterior ao HIV (G3) } & \multicolumn{2}{|c|}{ TB na notificação AIDS (G4) } & \multirow[t]{2}{*}{ Valor de $p$} \\
\hline & $n=639$ & $\%$ & $n=601$ & $\%$ & \\
\hline \multicolumn{6}{|l|}{ Sexo } \\
\hline Masculino & 464 & 72,6 & 428 & 71,2 & 0,584 \\
\hline Feminino & 175 & 27,4 & 173 & 28,8 & \\
\hline \multicolumn{6}{|l|}{ Faixa etária (anos) } \\
\hline $13-24$ & 119 & 18,6 & 89 & 14,8 & 0,001 \\
\hline $25-40$ & 375 & 58,7 & 322 & 53,6 & \\
\hline$\geq 40$ & 145 & 22,7 & 190 & 31,6 & \\
\hline \multicolumn{6}{|l|}{ Raça/Cor } \\
\hline Outras & 101 & 15,8 & 98 & 16,3 & 0,810 \\
\hline Parda & 538 & 84,2 & 503 & 83,7 & \\
\hline \multicolumn{6}{|l|}{ Categoria de transmissão } \\
\hline Homossexual/Bissexual & 196 & 30,7 & 123 & 20,5 & $<0,001$ \\
\hline Heterossexual & 300 & 47,0 & 326 & 54,2 & \\
\hline Sangue/Droga intravenosa & 36 & 5,6 & 12 & 2,0 & \\
\hline Ignorado & 107 & 16,7 & 140 & 23,3 & \\
\hline \multicolumn{6}{|l|}{ Residência } \\
\hline Capital & 577 & 90,3 & 547 & 91,0 & 0,665 \\
\hline Interior & 62 & 9,7 & 54 & 9,0 & \\
\hline \multicolumn{6}{|l|}{ Forma da TB } \\
\hline Pulmonar & 398 & 62,3 & 356 & 59,2 & 0,272 \\
\hline Extrapulmonar & 241 & 37,7 & 245 & 40,8 & \\
\hline \multicolumn{6}{|l|}{ Óbito } \\
\hline Sim & 187 & 29,3 & 186 & 30,9 & 0,518 \\
\hline Não & 452 & 70,7 & 415 & 69,1 & \\
\hline
\end{tabular}

Fonte: Sistema de Informação de Agravos de Notificação (SINAN) e Sistema de Informações sobre Mortalidade (SIM)/Amazonas.

cas com estar notificado para AIDS, somente encontrou associação com internação recorrente no mesmo hospital 20.

Chamou a atenção o alto número de casos que apresentaram TB depois de já se saberem portadores do HIV (G3), apesar das recomendações nacionais 7 e internacionais 8 para a prevenção de TB em PVHIV, que incluem o diagnóstico precoce de HIV na população em geral, seguido de avaliação imediata da presença de TB ativa e, excluída esta, avaliação de ILTB ${ }^{21}$, para que o tratamento desta última com isoniazida, associada ou não ao uso de ART 3,4,5 possa reduzir a morbimortalidade pela associação das duas doenças.

Este estudo apresenta limitações comuns aos que utilizam bases de dados secundárias, em que a acurácia e a completude das informações podem ser limitadoras dos achados. Entretanto, o relacionamento de bases de dados de sistemas de informação diferentes pode ajudar a qualificar as bases envolvidas, completando dados que trazem maior precisão aos estudos. No caso específico da base do SINAN AIDS, a variável categoria de exposição classicamente tem um preenchimento expressivo como ignorado 22, tendo o mesmo ocorrido com os dados do Amazonas, o que pode ter trazido alguma imprecisão ao se analisar esta variável. 


\section{Tabela 3}

Regressão logística univariada e multivariada das características dos casos de coinfecção tuberculose (TB)/HIV/AIDS, encontrados no Sistema de Informação de Agravos de Notificação (SINAN) AIDS, em relação à notificação ou não no SINAN TB. Amazonas, Brasil, 2001 a 2012.

\begin{tabular}{|c|c|c|c|c|c|c|}
\hline Características & OR bruta & IC95\% & Valor de $p$ & OR ajustada & IC95\% & Valor de $p$ \\
\hline Sexo masculino & 1,18 & $0,88-1,58$ & 0,278 & & & \\
\hline Cor parda & 1,12 & $0,82-1,54$ & 0,480 & & & \\
\hline \multicolumn{7}{|l|}{ Faixa etária (anos) } \\
\hline $13-24$ & 1,00 & & & & & \\
\hline $25-39$ & 1,16 & $0,80-1,17$ & 0,437 & & & \\
\hline$\geq 40$ & 1,31 & $0,86-1,98$ & 0,208 & & & \\
\hline Residente na capital & 1,75 & $1,15-2,66$ & 0,009 & 1,73 & $1,13-2,65$ & 0,011 \\
\hline \multicolumn{7}{|l|}{ Categoria de transmissão } \\
\hline Ignorado & 1,00 & & & & & \\
\hline Homo/bissexual & 0,90 & $0,60-1,36$ & 0,631 & & & \\
\hline Heterossexual & 1,12 & $0,80-1,56$ & 0,504 & & & \\
\hline Sangue/Droga intravenosa & 0,56 & $0,35-9,15$ & 0,687 & & & \\
\hline Óbito & 1,40 & $1,05-1,87$ & 0,024 & 1,41 & $1,05-1,88$ & 0,022 \\
\hline
\end{tabular}

IC95\%: intervalo de 95\% de confiança; OR: odds ratio.

Fonte: Sistema de Informação de Agravos de Notificação (SINAN) e Sistema de Informações sobre Mortalidade (SIM)/Amazonas.

\section{Tabela 4}

Regressão logística univariada e multivariada das características dos casos de coinfecção tuberculose (TB)/HIV/AIDS encontrados no Sistema de Informação de Agravos de Notificação (SINAN) TB, em relação à notificação ou não no SINAN AIDS. Amazonas, Brasil, 2001 a 2012.

\begin{tabular}{|c|c|c|c|c|c|c|}
\hline Características & OR bruta & IC95\% & Valor de p & OR ajustada & IC95\% & Valor de $p$ \\
\hline Sexo masculino & 1,07 & $0,88-1,31$ & 0,475 & & & \\
\hline Cor parda & 1,52 & $1,23-1,87$ & $<0,001$ & 1,67 & $1,35-2,08$ & $<0,001$ \\
\hline \multicolumn{7}{|l|}{ Faixa etária (anos) } \\
\hline $13-24$ & 1,00 & & & & & \\
\hline $25-39$ & 1,33 & $1,02-1,73$ & 0,032 & 1,23 & $1,03-1,48$ & $<0,001$ \\
\hline$\geq 40$ & 1,10 & $0,83-1,46$ & 0,488 & & & \\
\hline Residente na capital & 2,02 & $1,56-2,62$ & $<0,001$ & 2,05 & $1,58-2,67$ & $<0,001$ \\
\hline \multicolumn{7}{|l|}{ Forma da TB } \\
\hline Extrapulmonar & 1,00 & & & & & \\
\hline Pulmonar & 0,88 & $0,73-1,05$ & 0,155 & & & \\
\hline Óbito & 1,67 & $1,36-2,06$ & $<0,001$ & 1,77 & $1,43-2,19$ & $<0,001$ \\
\hline
\end{tabular}

IC95\%: intervalo de 95\% de confiança; OR: odds ratio.

Fonte: Sistema de Informação de Agravos de Notificação (SINAN) e Sistema de Informações sobre Mortalidade (SIM)/Amazonas. 


\section{Conclusões}

A prevalência estimada de coinfecção TB/HIV/AIDS no Estado do Amazonas foi de 7,7\% pelo relacionamento das bases de dados do SINAN AIDS e TB, enfatizando a importância do HIV como fator isolado para a ocorrência da TB nesta população. Do ponto de vista clínico, o grupo de pacientes em que a TB ocorreu após terem sido identificados como PVHIV foi o mais representativo, mesmo existindo recomendações para a redução da TB nesta população, o que colaboraria para a diminuição da mortalidade pela coinfecção.

Do ponto de vista da vigilância epidemiológica, a subnotificação de TB entre casos de AIDS foi de $35 \%$, um valor alto e que merece consideração. Por outro lado, o desconhecimento dos critérios para a notificação de casos de AIDS (e não de HIV-positivo) pode ter influenciado o não registro de 19,6\% de casos de TB/HIV como AIDS. A introdução da notificação compulsória de HIV a partir de 2014 deve melhorar esse cenário, mas a divulgação dos critérios de notificação de qualquer agravo deve fazer parte da educação permanente dos profissionais de saúde.

O linkage periódico dessas bases de dados pode ser uma ferramenta programática poderosa para diminuir a subnotificação, auxiliando no gerenciamento das duas endemias, permitindo a qualificação de todas as bases.

\section{Colaboradores}

E. S. Magno participou do desenho do estudo, análise e interpretação dos dados e preparo do artigo. V. Saraceni participou do desenho do estudo, análise e interpretação dos dados e preparo e revisão crítica do artigo. A. B. Souza participou da coleta e interpretação dos dados e preparo e revisão crítica do artigo. R. S. Magno e M. G. G. Saraiva participaram da interpretação dos dados e preparo e revisão do artigo. S. Bührer-Sékula participou do desenho do estudo, interpretação dos dados e preparo e revisão crítica do artigo.

\section{Agradecimentos}

As autoras agradecem a colaboração do Diretor-Presidente da Fundação de Vigilância e Saúde (FVS-AM) Dr. Bernadino Claúdio de Albuquerque; da Chefe/Gerente Alzira Cabrinha, do Núcleo de Sistema de Informação (NUSI); e Dra. Marlucia da Silva Garrido, Coordenadora do Programa Estadual de Controle da Tuberculose (Amazonas); à Fundação de Medicina Tropical Dr. Heitor Vieira Dourado; a Cristiane Benevides, responsável-técnica da Vigilância Epidemiológica da Coordenação Estadual de DST e AIDS do Estado do Amazonas pelo apoio logístico e técnico. À Fundação de Amparo à Pesquisa do Estado do Amazonas (FAPEAM) pelo financiamento.

\section{Referências}

1. Pawlowski A, Jansson M, Sköld M, Rottenberg ME, Källenius G. Tuberculosis and HIV coinfection. PLoS Pathog 2012; 8:e1002464.

2. Saraceni V, Durovni B, Cavalcante SC, Cohn S, Pacheco AG, Moulton LH, et al. Survival of HIV patients with tuberculosis started on simultaneous or deferred HAART in the THRio cohort, Rio de Janeiro, Brazil. Braz J Infect Dis 2014; 18:491-5.

3. Golub JE, Saraceni V, Cavalcante SC, Pacheco AG, Moulton LH, King BS, et al. The impact of antiretroviral therapy and isoniazid preventive therapy on tuberculosis incidence in HIV-infected patients in Rio de Janeiro, Brazil. AIDS 2007; 21:1441-8.

4. Santoro-Lopes G, de Pinho AMF, Harrison LH, Schechter M. Reduced risk of tuberculosis among Brazilian patients with advanced human immunodeficiency virus infection treated with highly active antiretroviral therapy. Clin Infect Dis 2002; 34:543-6.

5. Durovni B, Saraceni V, Moulton LH, Pacheco AG, Cavalcante SC, King BS, et al. Effect of improved tuberculosis screening and isoniazid preventive therapy on incidence of tuberculosis and death in patients with HIV in clinics in Rio de Janeiro, Brazil: a stepped wedge, cluster-randomised trial. Lancet Infect Dis 2013; 13:852-8. 
6. Grangeiro A, Escuder MM, Menezes PR, Alencar R, Ayres de Castilho E. Late entry into HIV care: estimated impact on AIDS mortality rates in Brazil, 2003-2006. PLoS One 2011; 6:p. e14585.

7. Comissão de Tuberculose da SBPT; Grupo de Trabalho das Diretrizes para Tuberculose da SBPT. III diretrizes da Associação Brasileira do Tórax em Tuberculose. J Bras Pneumol 2009; 35:1018-48.

8. World Health Organization. Consolidated guidelines on the use of antiretroviral drugs for treating and preventing HIV infection. http://apps.who.int/iris/bitstre am/10665/85321/1/9789241505727_eng.pdf (acessado em 30/Jun/2013).

9. Ministério da Saúde. Boletim Epidemiológico Tuberculose 2015. Detectar, tratar e curar: desafios e estratégias brasileiras frente à tuberculose. http://u.saude.gov.br/images/pdf/2015/ marco/25/Boletim-tuberculose-2015.pdf.

10. Saraceni V, King BS, Cavalcante SC, Golub JE, Lauria LM, Moulton LH, et al. Tuberculosis as primary cause of death among AIDS cases in Rio de Janeiro, Brazil. Int J Tuberc Lung Dis 2008; 12:769-72.

11. Souza SL, Feitoza PV, Araújo JR, Andrade RV, Ferreira LC. Causes of death among patients with acquired immunodeficiency syndrome autopsied at the Tropical Medicine Foundation of Amazonas. Rev Soc Bras Med Trop 2008; 41:247-55.

12. Secretaria de Vigilância em Saúde, Ministério da Saúde. Critérios de definição de casos de Aids em crianças e adultos. Brasília: Ministério da Saúde; 2004. (Série Manuais, 60).

13. Ministério da Saúde. Portaria MS/GM no 1.271, de 6 de junho de 2014. Diário Oficial da União 2014; 9 jun.

14. Camargo Jr. KR, Coeli CM. Reclink: aplicativo para o relacionamento de banco de dados implementando o método probabilistic record linkage. Cad Saúde Pública 2000; 16:439-47.
15. Carvalho CN, Dourado I, Bierrenbach AL. Underreporting of the tuberculosis and AIDS comorbidity: an application of the linkage method. Rev Saúde Pública 2011; 45:548-55.

16. Pinheiro RS, Andrade VL, Oliveira GP. Subnotificação da tuberculose no Sistema de Informação de Agravos de Notificação (SINAN): abandono primário de bacilíferos e captação de casos em outras fontes de informação usando linkage probabilístico. Cad Saúde Pública 2012; 28:1559-68.

17. Melosini L, Vetrano U, Dente FL, Cristofano M, Giraldi M, Gabbrielli L, et al. Evaluation of underreporting tuberculosis in Central Italy by means of record linkage. BMC Public Health 2012; 12:472

18. Oliveira GP, Pinheiro RS, Coeli CM, Barreira D, Codenotti SB. Mortality information system for identifying underreported cases of tuberculosis in Brazil. Rev Bras Epidemiol 2012; 15:468-77.

19. Ferreira VMB, Portela MC. Avaliação da subnotificação de casos de Aids no Município do Rio de Janeiro com base em dados do Sistema de Informações Hospitalares do Sistema Único de Saúde. Cad Saúde Pública 1999; 15:317-24.

20. Ferreira VM, Portela MC, Vasconcellos MT. Variables associated with under reporting of AIDS patients in Rio de Janeiro, Brazil, 1996. Rev Saúde Pública 2000; 34:170-7.

21. Jamal LF, Moherdaui F. Tuberculosis and HIV infection in Brazil: magnitude of the problem and strategies for control. Rev Saúde Pública 2007; 41:104-10.

22. Ministério da Saúde. Proporção de casos de aids por categoria de exposição - D.31. http:// tabnet.datasus.gov.br/tabdata/livroidb/ idb2010/d31.pdf. 


\section{Abstract}

Tuberculosis $(T B)$ in persons living with HIV (PL$H I V)$ is the leading infectious cause of AIDS-related death. The aim of this study was to estimate the prevalence of $T B / H I V$ coinfection, evaluate notification of the two diseases over time by probabilistic database linkage, and identify factors associated with AIDS and TB notifications. Prevalence of TB/HIV coinfection was $7.7 \%$. The group of PLHIV with subsequent TB diagnosis was the most representative, despite available preventive measures. Underreporting of TB among AIDS cases was $35 \%$, and $19.6 \%$ of TB cases could have been reported as AIDS. For AIDS cases with mention of TB, living in the state capital showed $75 \%$ greater odds of being reported to the Tuberculosis Notification System (SINAN-TB), and having died increased the odds of reporting by 40\%. Of TB cases with mention of HIV, brown skin color, age 25 to 39 years, living in the state capital, and having evolved to death were associated with higher odds of reporting to the AIDS Notification System. Periodic linkage of these databases can be a powerful tool for programs to decrease underreporting.

Disease Notification; Opportunistic Infections; Tuberculosis; HIV

\section{Resumen}

La tuberculosis (TB) en personas viviendo con VIH (PVVIH) es la mayor causa infecciosa de muerte relacionada con el SIDA. El objetivo de este trabajo fue estimar la prevalencia de la coinfección TB/ VIH, evaluar la notificación de las dos enfermedades de manera temporal, relación de bases de datos, $y$ buscar factores asociados a las notificaciones de SIDA y TB. La prevalencia de coinfección TB/ VIH fue de un 7,7\%. El grupo de PVVIH, con posterior diagnóstico de TB, fue el más representativo, a pesar de las medidas preventivas disponibles. La subnotificación de TB entre los casos de SIDA fue de un 35\%, y un 19,6\% de los casos de TB podrían ser notificados como SIDA. Para los casos de SIDA con mención de $T B$, residir en la capital presentó una oportunidad un 75\% mayor de ser notificada en el Sistema de Notificación de Tuberculosis (SINAN TB), y haber fallecido aumentó en un 40\% la oportunidad de registro. De los casos de TB con mención de VIH, ser mestizo, tener entre 25 y 39 años, residir en la capital y haber evolucionado hacia el óbito estuvieron asociados a una mayor oportunidad de notificación en el SIDA. La vinculación periódica de estas bases puede ser una herramienta programática poderosa para disminuir la subnotificación.

Notificación de Enfermedad; Infecciones

Oportunistas; Tuberculosis. VIH
Recebido em 20/Fev/2015

Versão final reapresentada em 29/Abr/2016

Aprovado em 20/Jul/2016 